\title{
First report of a 'Candidatus Phytoplasma cynodontis'-related strain (group 16SrXIV) associated with Huanglongbing disease on Citrus grandis
}

\author{
D. K. Ghosh ${ }^{1} \cdot$ M. Motghare ${ }^{1} \cdot$ A. Kokane ${ }^{1} \cdot$ S. Kokane ${ }^{1} \cdot$ A. Warghane ${ }^{1} \cdot$ S. Bhose ${ }^{1} \cdot$ D. Surwase ${ }^{1} \cdot$ M. S. Ladaniya ${ }^{1}$
}

Received: 20 October 2018 / Accepted: 25 March 2019 / Published online: 1 April 2019

(C) Australasian Plant Pathology Society Inc. 2019

\begin{abstract}
Association of phytoplasma with Huanglongbing (HLB) disease is reported here for the first time in Citrus grandis from India. Molecular investigation of the pathogen reveals that it belongs to 16SrXIV Group of phytoplasma, 'Candidatus Phytoplasma cynodontis'. The association of phytoplasma with HLB accentuates that there is need to broaden the disease management strategies.
\end{abstract}

Keywords Phytoplasma $\cdot$ Huanglongbing $\cdot$ Citrus grandis $\cdot$ Yellow mottling

Huanglongbing (HLB) or citrus greening disease is one of the most destructive citrus diseases and causes extensive economic losses to the citrus industry in India and most of the citrus growing regions of the world (Ghosh et al. 2015). Although the disease is primarily associated with 'Candidatus Liberibacter spp.', a group of gram-negative, phloem-limited a-proteobacteria, there are some reports which also link HLB with phytoplasmas (Lou et al. 2014; Das et al. 2016). Citrus leaf samples showing typical HLB yellow mottling symptoms were found positive with phytoplasma belonging to different groups like 16SrIX in sweet orange from Brazil (Teixeira et al. 2008), 16SrI in mandarin, sweet orange and pummelo and 16SrII in grapefruit from China (Chen et al. 2009; Lou et al. 2014), and 16SrVI in mandarin from India (Das et al. 2016). To study the HLB association with phytoplasma in more cultivars from India, a survey was conducted during 2017-18 in Nagpur District of Maharashtra state. Leaf samples of 20 pummelo (Citrus grandis) plants showing yellow mottling symptoms were collected (Fig. 1). The collected samples were washed then wiped with $70 \%$ ethanol to avoid any surface contamination. Midribs and petioles were excised and ground in liquid nitrogen, and $100 \mathrm{mg}$ of the powdered sample was used for DNA extraction using the DNeasy Plant mini kit (Qiagen, Hilden, Germany) according to the manufacturer's

D. K. Ghosh

ghoshdk@hotmail.com

1 ICAR- Central Citrus Research Institute, Nagpur 440033, India protocol. The presence of 'Candidatus Liberibacter asiaticus' was tested using previously described polymerase chain reaction (PCR) amplification of 16S rRNA region with primer set OI1/OI2c (Garnier et al. 2000). To determine association with phytoplasma, the samples were analysed using P1/P7 universal primers specific to phytoplasmas for the first round of PCR and the products were subsequently amplified with nested primer pairs R16F2n/R16R2 and Fu5/Ru3 (Lee et al. 2004; Lou et al. 2014; Ghosh et al. 2017). Among 20 samples, 13 were found to be positive only for HLB and 2 were positive for both HLB and phytoplasma. The amplicons of expected band length, $\sim 1.2 \mathrm{~kb}$ for OI1/OI2c (Fig. 2), $1.2 \mathrm{~kb}$ for R16F2n/R16R (Fig. 3) and $\sim 0.88 \mathrm{~kb}$ for Fu5/Ru3 (Fig. 4) were observed on $1 \%$ agarose gel. The positive phytoplasma sample (DNA) used in the study was 'Candidatus Phytoplasma trifolii' (16SrVI group) donated by Dr. G P Rao, IARI, Delhi. The negative control was a healthy sample, free from the pathogens and maintained in an insect protected poly-house at ICAR-CCRI, Nagpur. Amplicons obtained with primer set R16F2n/R16R were purified, sequenced (Eurofins, Bangalore) and deposited in GenBank. Both the sequences were identical with each other. NCBI-BLAST analysis showed that the HLB phytoplasma isolate from Nagpur (GenBank accession number MK041924 and MK012544) shared the highest identity (99\%) with 'Candidatus Phytoplasma cynodontis' strains (LT558777, KM220612, AB741630). Phylogenetic analysis of 16S rRNA sequences was done using software MEGA 7.0 by maximum likelihood method with 1000 bootstrap value (Tamura and Nei 1993). The phylogenetic tree showed that 
Fig. 1 Pummelo plant showing typical citrus greening symptoms a yellow shoot and, b blotchy mottling
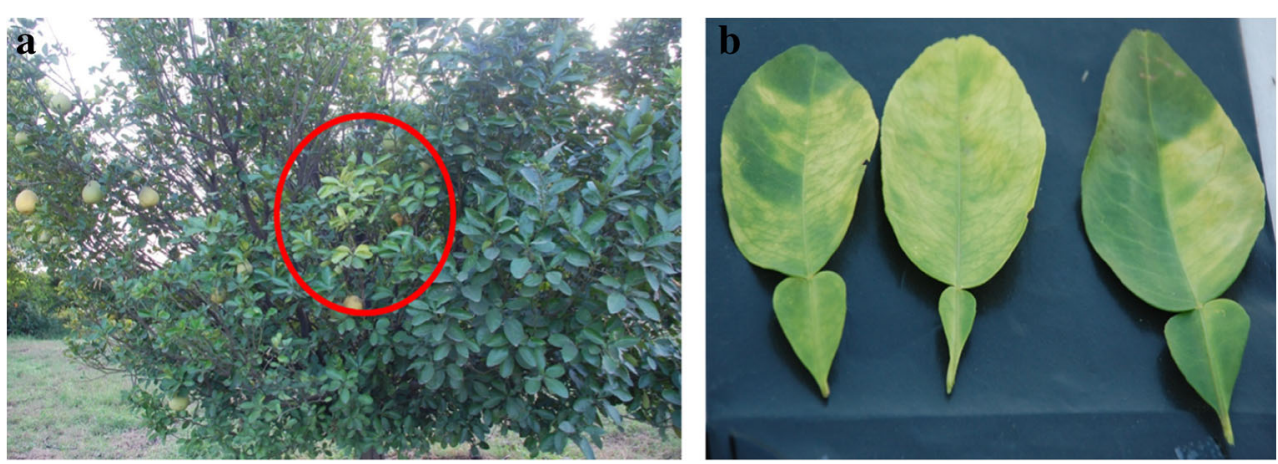

Fig. 2 Agarose gel photograph of PCR amplicons of pummelo samples with primer set OI1/ OI2c, Lane L: 100 bp DNA ladder, Lane 1-10: symptomatic samples, Lane 11: non template control, Lane 12: positive control, Lane 13-22: symptomatic samples, Lane 23: non template control, Lane 24: positive control

Fig. 3 Agarose gel photograph of PCR amplicons of pummelo samples with primer set R16F2n/ R16R2, Lane L: 1 kp DNA ladder, Lane 1-10: symptomatic samples, Lane 11: non template control, Lane 12: nested negative control, Lane 13: negative control, Lane 14: positive control, Lane 15-24: symptomatic samples, Lane 25: non template control, Lane 26: nested negative control, Lane 27: negative control, Lane 28: positive control
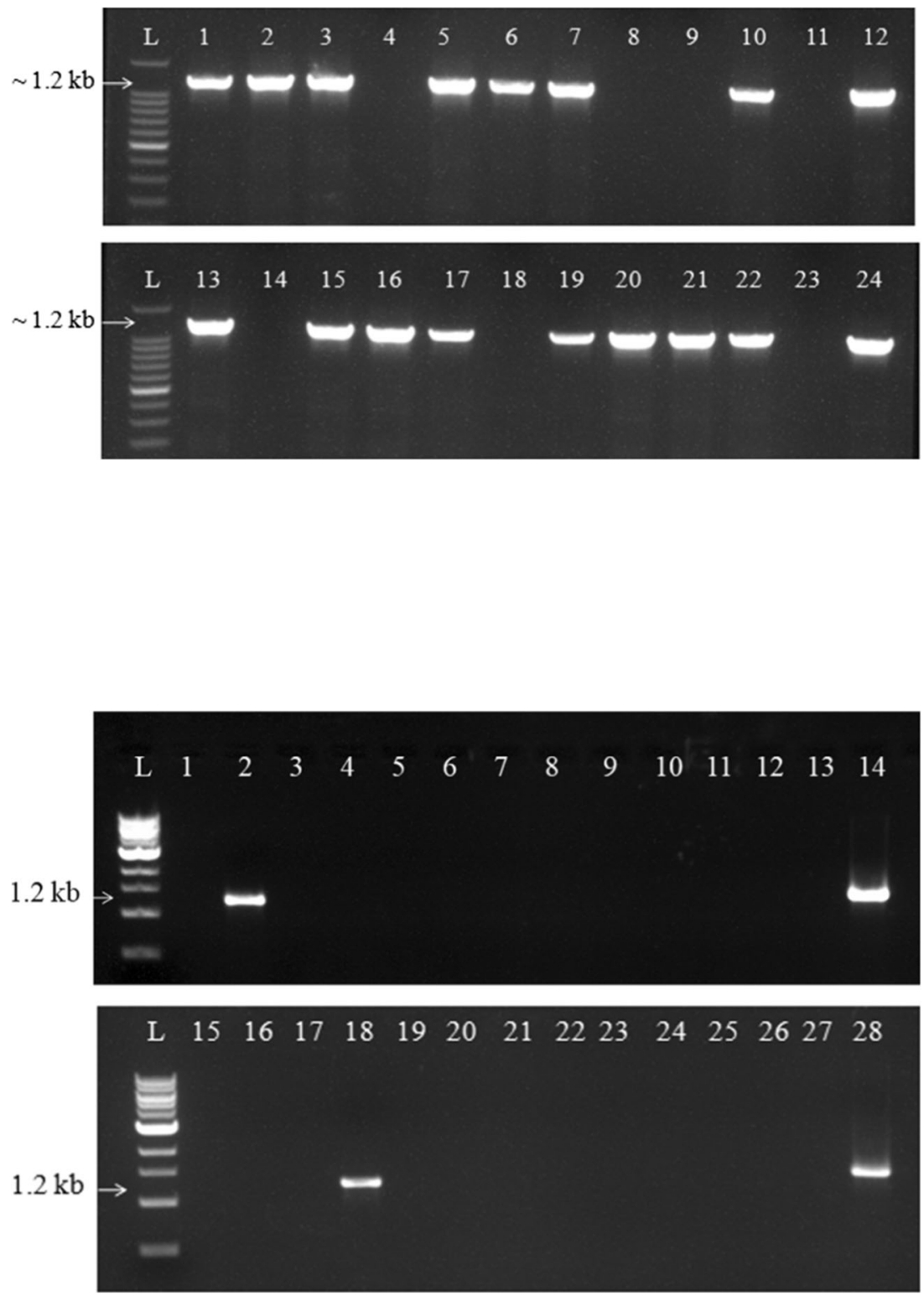
Fig. 4 Agarose gel photograph of PCR amplicons of pummelo samples with primer set Fu5/Ru3, Lane L: $1 \mathrm{kp}$ DNA ladder, Lane 1-10: symptomatic samples, Lane 11: non template control, Lane 12: nested negative control, Lane 13: negative control, Lane 14: positive control, Lane 15-24: symptomatic samples, Lane 25 : non template control, Lane 26 : nested negative control, Lane 27: negative control, Lane 28: positive control
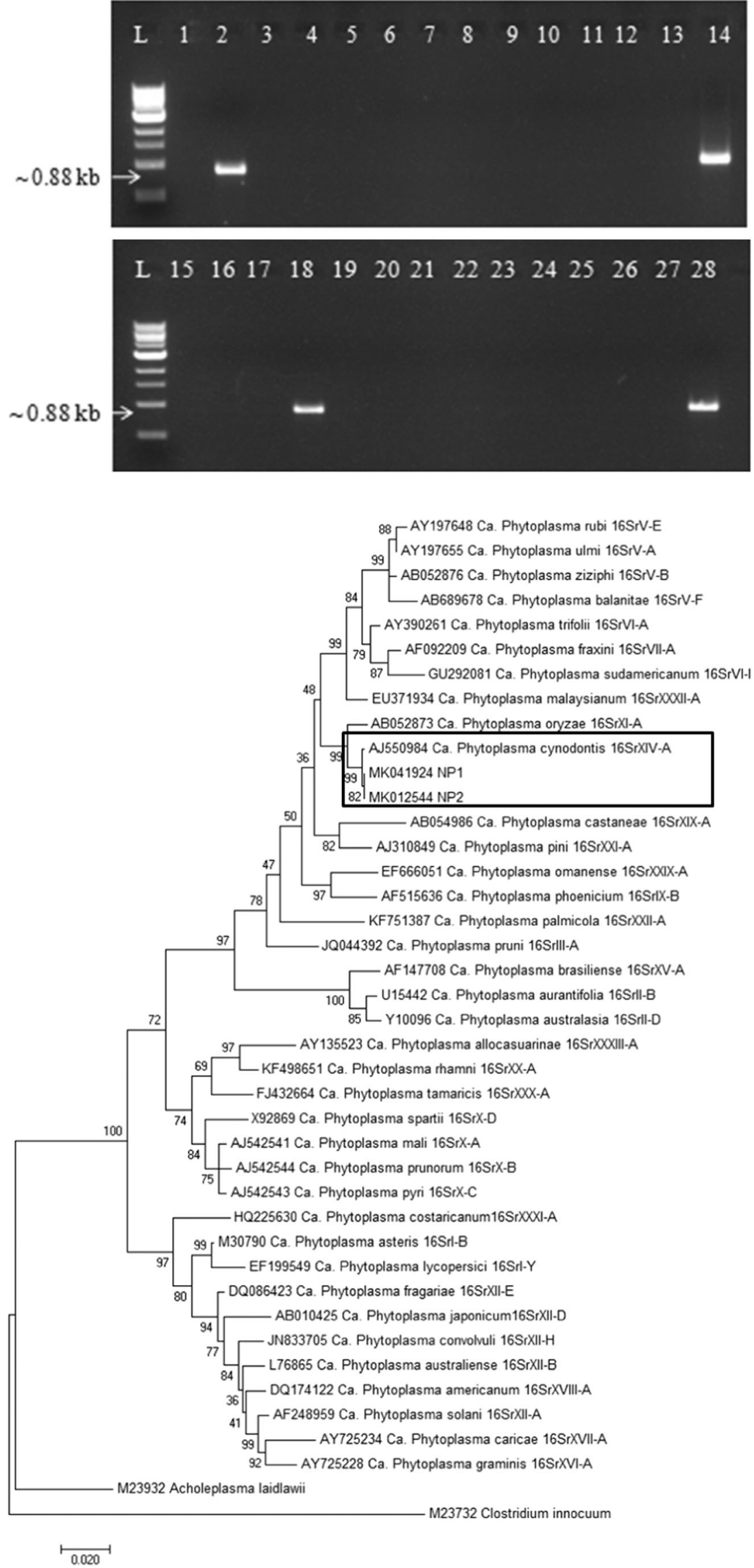

Fig. 5 Phylogenetic tree constructed by MEGA 7.0 software using $16 \mathrm{~s}$ rRNA sequences of NP1 and 2 HLBphytoplasma samples along with 37 groups and sub-groups of phytoplasma which were obtain from GenBank. The numbers on the branches indicates percentage of replicate trees in which the groups clustered together (1000 replicates) and Clostridium innocuum, Acholeplasma laidlawii used as outgroup 
the samples formed a distinct clade with 'Candidatus Phytoplasma cynodontis' isolate BGW1-C1 (Fig. 5). In-silico RFLP analysis of R16F2n/R16R2 sequence of sample NP1 was performed with 17 restriction enzymes (AluI, BamHI, BfaI, BstUI, DraI, EcoRI, HaeIII, HhaI, HinfI, HpaI, HpaII, KpnI, MseI, RsaI, Sau3AI, SspI and TaqI) using iPhyClassifier online tool (Zhao et al. 2009). The virtual RFLP pattern was found to be similar with the reference pattern of $16 \mathrm{Sr}$ group XIV, subgroup A (AJ550984). To the best of our knowledge, this is the first report of association of 'Candidatus Phytoplasma cynodontis' (16SrXIV) strain with HLB in India. It will be interesting to identify whether any other Phytoplasma groups are also associated with HLB, any synergistic effect of both 'Candidatus Liberibacter asiaticus' and 'Candidatus Phytoplasma cynodontis' on symptom expression in infected plant and also to identify any potential insect vectors and plant reservoirs in this region.

\section{References}

Chen J, Pu X, Deng X, Liu S, Li H, Civerolo E (2009) A phytoplasma related to 'Candidatus Phytoplasma asteris' detected in citrus showing huanglongbing (yellow shoot disease) symptoms in Guangdong, P. R. China. Phytopathology 99:236-242

Das AK, Nerkar S, Thakre N, Kumar A (2016) First report of 'Candidatus Phytoplasma trifolii' (16SrVI group) in Nagpur mandarin (Citrus reticulata) showing huanglongbing symptoms in Central India. New Dis Reports 34:15
Garnier M, Jagoueix-Eveillard S, Cronje PR, Le Roux HF, Bove JM (2000) Genomic characterization of a Liberibacter present in an ornamental rutaceous tree, Calodendrum capense, in the Western Cape Province of South Africa. Proposal of 'Candidatus Liberibacter africanus subsp. capensis. Int J Evol Microbiol 50: 2119-2125

Ghosh DK, Bhose S, Motghare M, Warghane A, Mukherjee K, Ghosh DK Sr, Sharma AK, Ladaniya MS, Gowda S (2015) Genetic diversity of the Indian populations of 'Candidatus liberibacter asiaticus' based on the tandem repeat variability in a genomic locus. Phytopathology 105:1043-1049

Ghosh DK, Bhose S, Sharma P, Warghane A, Motghare M, Ladaniya MS, Reddy MK, Thorat V, Yadav A (2017) First report of 16SrXIV group phytoplasma associated with witches'-broom disease of acid lime (Citrus auranifolia) in India. Plant Dis 101(5):831

Lee IM, Martini M, Marcone C, Zhu SF (2004) Clasification of phytoplasma strains in the elm yellows group (16SrV) and proposal of 'Candidatus Phytoplasma ulmi' for the phytoplasma associated with elm yellows. Int J Syst Evol Microbiol 54:337-347

Lou B, Bai X, Bai Y, Deng C, Roy Chowdhury M, Chen C, Song Y (2014) Detection and molecular characterization of a 16SrII-A* phytoplasma in grapefruit (Citrus paradisi) with huanglongbinglike symptoms in China. J Phytopathol 162:387-395

Tamura K, Nei M (1993) Estimation of the number of nucleotide substitutions in the control region of mitochondrial DNA in humans and chimpanzees. Mol Biol Evol 10:512-526

Teixeira DC, Wulff NA, Martins EC, Kitajima EW, Bassanezi R, Ayres AJ, Eveillard S, Saillard C, Bove JM (2008) A phytoplasma closely related to the pigeon pea witches'-broom phytoplasma (16Sr IX) is associated with citrus huanglongbing symptoms in the state of São Paulo, Brazil. Phytopathology 98:977-984

Zhao Y, Wei W, Lee IM, Shao J, Suo X, Davis RE (2009) Construction of an interactive online phytoplasma classification tool, iphyClassifier, and its application in analysis of the peach X-disease phytoplasma group (16SrIII). Int J Syst Evol Microbiol 59:2582-2593 